Korean J. Math. 22 (2014), No. 3, pp. 419-427

http://dx.doi.org/10.11568/kjm.2014.22.3.419

\title{
NOTE ON AVERAGE OF CLASS NUMBERS OF CUBIC FUNCTION FIELDS
}

\author{
HWANYUP JUNG
}

\begin{abstract}
Let $k=\mathbb{F}_{q}(T)$ be the rational function field over a finite field $\mathbb{F}_{q}$, where $q \equiv 1 \bmod 3$. In this paper, we determine asymptotic values of average of ideal class numbers of some family of cubic Kummer extensions of $k$.
\end{abstract}

\section{Introduction and statement of result}

Let $k=\mathbb{F}_{q}(T)$ be the rational function field over a finite field $\mathbb{F}_{q}$ and $\mathbb{A}=\mathbb{F}_{q}[T]$. Average values of $L$-functions associated to orders in quadratic extensions of $k$ are obtained by Hoffstein and Rosen [3] when $q$ is odd and by Chen [2] when $q$ is even. Rosen [6] generalized some results of [3] to general Kummer extensions of $k$ of degree $\ell$, where $\ell$ is a prime divisor of $q-1$, and determined average values of ideal class numbers of Kummer extensions of $k$ of degree 3. Prime [5] obtained an $L$-function average over imaginary quadratic extensions of $k$ with prime discriminants. Recently, Bae, Jung and Kang [1] extended the result of Chen to general Artin-Schreier extensions of $k$ and determined average values of ideal class numbers of Artin-Schreier extensions of $k$ of degree 3 . They also extended the result of Prime to general Kummer extensions of $k$ and obtained a similar formulas of average values of prime $L$-functions

Received May 5, 2014. Revised July 25, 2014. Accepted July 25, 2014.

2010 Mathematics Subject Classification: 11R58, 11R16, 11R29.

Key words and phrases: Average of class numbers, cubic function field.

This research was supported by Basic Science Research Program through the National Research Foundation of Korea(NRF) funded by the Ministry of Education(2010-0008139).

(c) The Kangwon-Kyungki Mathematical Society, 2014.

This is an Open Access article distributed under the terms of the Creative commons Attribution Non-Commercial License (http://creativecommons.org/licenses/by -nc/3.0/) which permits unrestricted non-commercial use, distribution and reproduction in any medium, provided the original work is properly cited. 
of Artin-Schreier extensions of $k$ of degree 2. The aim of this paper is to determine asymptotic values of average of ideal class numbers of some family of cubic Kummer extensions of $k$.

To state our main result, we introduce some notations. Assume that $q \equiv 1 \bmod 3$. A monic irreducible polynomial in $\mathbb{A}$ will be called a prime polynomial. Any (geometric) cubic Kummer extension $K$ of $k$ can be written as $K=k(\sqrt[3]{D})$ for some cubic power free polynomial $D \in \mathbb{A}$. We say that $K / k$ is ramified imaginary, real or inert imaginary according as the infinite prime $\infty_{k}$ of $k$ ramifies, splits completely or is inert in $K$. Let $\mathcal{O}_{K}$ be the integral closure of $\mathbb{A}$ in $K$. Write $h\left(\mathcal{O}_{K}\right), d\left(\mathcal{O}_{K}\right)$ and $R\left(\mathcal{O}_{K}\right)$ for the ideal class number, the discriminant and the regulator of $\mathcal{O}_{K}$, respectively. For $K=k(\sqrt[3]{D})$, we have $d\left(\mathcal{O}_{K}\right)=\operatorname{rad}(D)^{2}$ (see $(2.1))$, where $\operatorname{rad}(D)$ is the product of distinct prime divisors of $D$, and $R\left(\mathcal{O}_{K}\right)=1$ if $K / k$ is imaginary. Let $\mathcal{A}$ be the family of all ramified imaginary cubic Kummer extensions $K$ of $k$ such that $d\left(\mathcal{O}_{K}\right)$ is a square of a prime polynomial and $\mathcal{A}_{n}=\left\{K \in \mathcal{A}: \operatorname{deg} d\left(\mathcal{O}_{K}\right)=2 n\right\}$ for each positive integer $n$ with $3 \nmid n$. Similarly, replacing "ramified imaginary" by "real" or "inert imaginary" and " $3 \nmid n$ " by " $3 \mid n$ ", we define $\mathcal{B}, \mathcal{B}_{n}$ or $\mathcal{C}, \mathcal{C}_{n}$, respectively. We determine asymptotic values of average of ideal class numbers $h\left(\mathcal{O}_{K}\right)$ (or times regulator $R\left(\mathcal{O}_{K}\right)$ ) as $K$ varies over $\mathcal{A}, \mathcal{B}$, or $\mathcal{C}$. Our main result is the following theorem.

Theorem 1.1. Assume that $q \equiv 1 \bmod 3$. Then we have

1. as $n \rightarrow \infty$ with $3 \nmid n$,

$$
\frac{1}{\# \mathcal{A}_{n}} \sum_{K \in \mathcal{A}_{n}} h\left(\mathcal{O}_{K}\right)=\frac{\zeta_{\mathbb{A}}(2) \zeta_{\mathbb{A}}(3)^{2}}{\zeta_{\mathbb{A}}(6)} q^{n-1}+O\left(4^{n} q^{\frac{n}{2}}\right)
$$

2. as $n \rightarrow \infty$ with $3 \mid n$,

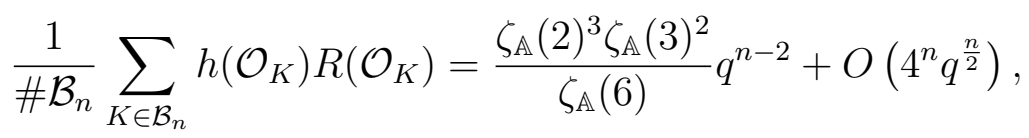

3. as $n \rightarrow \infty$ with $3 \mid n$,

$$
\frac{1}{\# \mathcal{C}_{n}} \sum_{K \in \mathcal{C}_{n}} h\left(\mathcal{O}_{K}\right)=\frac{3 \zeta_{\mathbb{A}}(3)^{2} \zeta_{\mathbb{A}}(4)}{\zeta_{\mathbb{A}}(6)} q^{n-2}+O\left(4^{n} q^{\frac{n}{2}}\right)
$$

where $\zeta_{\mathbb{A}}(s)=\frac{1}{1-q^{1-s}}$ is the zeta function of $\mathbb{A}$. 


\section{2. $L$-functions of Kummer extensions and Class number for- mula}

In this section we recall some basic results on Kummer extensions of $k$. For more details, we refer to $[6, \S 1]$. Let $\mathbb{A}^{+}$be the subset of $\mathbb{A}$ consisting of all monic polynomials and $\mathcal{P}(\mathbb{A})$ be the set of prime polynomials in $\mathbb{A}$. Let $\mathbb{A}_{n}^{+}=\left\{N \in \mathbb{A}^{+}: \operatorname{deg} N=n\right\}$ and $\mathcal{P}_{n}(\mathbb{A})=\mathcal{P}(\mathbb{A}) \cap \mathbb{A}_{n}^{+}$for $n \geq 1$. Assume that $q$ is a power of an odd prime. Let $\ell$ be a prime divisor of $q-1$. For an $\ell$-th power free polynomial $D \in \mathbb{A}$, let $\mathcal{O}_{K}$ be the integral closure of $\mathbb{A}$ in $K=k(\sqrt[\ell]{D})$. Let $h\left(\mathcal{O}_{K}\right), d\left(\mathcal{O}_{K}\right)$ and $R\left(\mathcal{O}_{K}\right)$ be the ideal class number, the discriminant and the regulator of $\mathcal{O}_{D}$, respectively. By [6, Theorem 1.2], we have

$$
d\left(\mathcal{O}_{K}\right)=\operatorname{rad}(D)^{\ell-1}
$$

where $\operatorname{rad}(D)$ is the product of distinct prime divisors of $D$. The decomposition of the infinite prime $\infty_{k}$ of $k$ in $K=k(\sqrt[\ell]{D})$ is determined as follows:

- $\infty_{k}$ ramifies in $K$ if and only if $\ell \nmid \operatorname{deg} D$. In this case $K / k$ is called a ramified imaginary extension.

- $\infty_{k}$ splits completely in $K$ if and only if $\ell \mid \operatorname{deg} D$ with $\operatorname{sgn}(D) \in$ $\mathbb{F}_{q}^{* \ell}$. In this case $K / k$ is called a real extension.

- $\infty_{k}$ is inert in $K$ if and only if $\ell \mid \operatorname{deg} P$ with $\operatorname{sgn}(D) \notin \mathbb{F}_{q}^{* \ell}$. In this case $K / k$ is called an inert imaginary extension.

We note that $R_{\mathcal{O}_{K}}=1$ if $K / k$ is imaginary. To defined the character $\chi_{D}$, we first need to fix an isomorphism $\omega$ between the group of $\ell$-th roots of unity in $\mathbb{C}$ and the group of $\ell$-th roots of unity in $\mathbb{F}_{q}$. For a prime polynomial $P \in \mathcal{P}(\mathbb{A})$, define $\chi_{D}(P)=0$ if $P \mid D$, and if $P \nmid D$, $\chi_{D}(P) \in \mathbb{C}^{*}$ is defined by

$$
D^{\frac{|P|-1}{\ell}} \equiv \omega\left(\chi_{D}(P)\right) \bmod P .
$$

Now we extend the definition to all of $\mathbb{A}^{+}$by multiplicativity. Then the $L$-function $L\left(s, \chi_{D}^{i}\right)$ associated to $\chi_{D}^{i}(0 \leq i \leq \ell-1)$ is defined by

$$
L\left(s, \chi_{D}^{i}\right)=\sum_{N \in \mathbb{A}^{+}} \frac{\chi_{D}^{i}(N)}{|N|^{s}} .
$$


It is well known $\left(\left[6\right.\right.$, Lemma 2.1]) that $L\left(s, \chi_{D}^{i}\right)$ is a polynomial in $q^{-s}$ of degree at most $\operatorname{deg} D-1$ for $1 \leq i \leq \ell-1$. Thus, we can write

$$
L\left(s, \chi_{D}^{i}\right)=\sum_{n=0}^{\operatorname{deg} D-1} \sum_{N \in \mathbb{A}_{n}^{+}} \chi_{D}^{i}(N) q^{-n s}
$$

For $K=k(\sqrt[3]{D})$, we have the following class number formula (see [6, Theorem 1.3]):

$$
\prod_{i=1}^{\ell-1} L\left(1, \chi_{D}^{i}\right)= \begin{cases}q^{\frac{\ell-1}{2}} \frac{h\left(\mathcal{O}_{K}\right)}{\sqrt{\left|d\left(\mathcal{O}_{K}\right)\right|}} & \text { if } K / k \text { is ramified imaginary } \\ (q-1)^{\ell-1} \frac{h\left(\mathcal{O}_{K}\right) R\left(\mathcal{O}_{K}\right)}{\sqrt{\left|d\left(\mathcal{O}_{K}\right)\right|}} & \text { if } K / k \text { is real } \\ \frac{q^{\ell}-1}{\ell(q-1)} \frac{h\left(\mathcal{O}_{K}\right)}{\sqrt{\left|d\left(\mathcal{O}_{K}\right)\right|}} & \text { if } K / k \text { is inert imaginary. }\end{cases}
$$

\section{Proof of Theorem 1.1}

In this section we give a proof of Theorem 1.1. Assume that $q \equiv$ $1 \bmod 3$. Let $\gamma$ be a generator of $\mathbb{F}_{q}^{*}$ and $\gamma_{j}=\gamma^{j}$ for $j \geq 0$. Then $\left\{\gamma_{0}=1, \gamma_{1}, \gamma_{2}\right\}$ forms a complete set of representatives of $\mathbb{F}_{q}^{*} / \mathbb{F}_{q}^{* 3}$. By Kummer theory, it is easy to see that any cubic Kummer extension $K$ of $k$ such that the discriminant $d\left(\mathcal{O}_{K}\right)$ is a square of a prime polynomial can be written uniquely as $K=k\left(\sqrt[3]{\gamma_{j} P}\right)$ with $P \in \mathcal{P}(\mathbb{A})$ and $0 \leq j \leq 2$. For $K=k\left(\sqrt[3]{\gamma_{j} P}\right)$, by $(2.1)$, we have $d\left(\mathcal{O}_{K}\right)=P^{2}$ and $K / k$ is ramified imaginary, splits completely or inert imaginary according as $3 \nmid \operatorname{deg} P$, $3 \mid \operatorname{deg} P$ with $j=0$ or $3 \mid \operatorname{deg} P$ with $j=1,2$, respectively. Moreover, by $(2.2)$, we have

$$
\prod_{i=1}^{2} L\left(1, \chi_{\gamma_{j} P}^{i}\right)= \begin{cases}q^{1-\operatorname{deg} P} h\left(\mathcal{O}_{K}\right) & \text { if } 3 \nmid \operatorname{deg} P, \\ (q-1)^{2} q^{-\operatorname{deg} P} h\left(\mathcal{O}_{K}\right) R\left(\mathcal{O}_{K}\right) & \text { if } 3 \mid \operatorname{deg} P \text { and } j=0, \\ \frac{q^{-\operatorname{deg} P}\left(q^{3}-1\right)}{3(q-1)} h\left(\mathcal{O}_{K}\right) & \text { if } 3 \mid \operatorname{deg} P \text { and } j=1,2 .\end{cases}
$$

Hence, for a positive integer $n$ with $3 \nmid n$, we have

$$
\mathcal{A}_{n}=\left\{k\left(\sqrt[3]{\gamma_{j} P}\right): P \in \mathcal{P}_{n}(\mathbb{A}), 0 \leq j \leq 2\right\}
$$


and

$$
\frac{1}{\# \mathcal{A}_{n}} \sum_{K \in \mathcal{A}_{n}} h\left(\mathcal{O}_{K}\right)=\frac{1}{3 \# \mathcal{P}_{n}(\mathbb{A})} \sum_{P \in \mathcal{P}_{n}(\mathbb{A})} \sum_{j=0}^{2} h\left(\mathcal{O}_{k\left(\sqrt[3]{\gamma_{j} P}\right)}\right) .
$$

For a positive integer $n$ with $3 \mid n$, we have

$$
\mathcal{B}_{n}=\left\{k(\sqrt[3]{P}): P \in \mathcal{P}_{n}(\mathbb{A})\right\}, \quad \mathcal{C}_{n}=\left\{k\left(\sqrt[3]{\gamma_{j} P}\right): P \in \mathcal{P}_{n}(\mathbb{A}), j=1,2\right\}
$$

$$
\frac{1}{\# \mathcal{B}_{n}} \sum_{K \in \mathcal{B}_{n}} h\left(\mathcal{O}_{K}\right) R\left(\mathcal{O}_{K}\right)=\frac{1}{\# \mathcal{P}_{n}(\mathbb{A})} \sum_{P \in \mathcal{P}_{n}(\mathbb{A})} h\left(\mathcal{O}_{k(\sqrt[3]{P})}\right) R\left(\mathcal{O}_{k(\sqrt[3]{P})}\right),
$$

and

$$
\frac{1}{\# \mathcal{C}_{n}} \sum_{K \in \mathcal{C}_{n}} h\left(\mathcal{O}_{K}\right)=\frac{1}{2 \# \mathcal{P}_{n}(\mathbb{A})} \sum_{P \in \mathcal{P}_{n}(\mathbb{A})} \sum_{j=1}^{2} h\left(\mathcal{O}_{k\left(\sqrt[3]{\gamma_{j} P}\right)}\right)
$$

We need a lemma which will be used in the proof of Proposition .

Lemma 3.1. For any positive integer $n, D \in \mathbb{A}^{+}$not cubic power and $i=1,2$, we have

$$
\left|\sum_{P \in \mathcal{P}_{n}(\mathbb{A})} \chi_{\gamma_{j} P}^{i}(D)\right| \leq \frac{(\operatorname{deg} D+1)}{n} q^{\frac{n}{2}} .
$$

In particular, if $\operatorname{deg} D<n$, then

$$
\left|\sum_{P \in \mathcal{P}_{n}(\mathbb{A})} \chi_{\gamma_{j} P}^{i}(D)\right| \leq 2 q^{\frac{n}{2}}
$$

Proof. (3.5) follows from Theorem 2.1 in [4] and cubic power reciprocity law. For (3.6), by (3.5), we have

$$
\left|\sum_{P \in \mathcal{P}_{n}(\mathbb{A})} \chi_{\gamma_{j} P}^{i}(D)\right| \leq\left(1+\frac{1}{n}\right) q^{\frac{n}{2}} \leq 2 q^{\frac{n}{2}}
$$

For a positive integer $n$, let

$$
\mathcal{Z}_{n, j}(s)=\frac{1}{\# \mathcal{P}_{n}(\mathbb{A})} \sum_{P \in \mathcal{P}_{n}(\mathbb{A})} L\left(s, \chi_{\gamma_{j} P}\right) L\left(s, \chi_{\gamma_{j} P}^{2}\right) \quad(0 \leq j \leq 2)
$$


Proposition 3.2. We have

$$
\mathcal{Z}_{n, j}(1)=\frac{1}{\# \mathcal{P}_{n}(\mathbb{A})} \sum_{P \in \mathcal{P}_{n}(\mathbb{A})} \prod_{i=1}^{2} L\left(1, \chi_{\gamma_{j} P}^{i}\right)=\frac{\zeta_{\mathbb{A}}(2) \zeta_{\mathbb{A}}(3)^{2}}{\zeta_{\mathbb{A}}(6)}+O\left(4^{n} q^{-\frac{n}{2}}\right) .
$$

Proof. Since $L\left(s, \chi_{\gamma_{j} P}\right)$ and $L\left(s, \chi_{\gamma_{j} P}^{2}\right)$ are polynomials in $q^{-s}$ of degree $\leq n-1$, we can write

$$
\prod_{i=1}^{2} L\left(s, \chi_{\gamma_{j} P}^{i}\right)=\sum_{m=0}^{2 n-2} \sum_{\substack{m_{1}+m_{2}=m \\\left(M_{1}, M_{2}\right) \in \mathbb{A}_{m_{1}}^{+} \times \mathbb{A}_{m_{2}}^{+}}} \chi_{\gamma_{j} P}\left(M_{1} M_{2}^{2}\right) q^{-m s}
$$

Thus,

$$
\mathcal{Z}_{n, j}(s)=\frac{1}{\# \mathcal{P}_{n}(\mathbb{A})} \sum_{m=0}^{2 n-2} \sum_{P \in \mathcal{P}_{n}(\mathbb{A})} a_{m}\left(\gamma_{j} P\right) q^{-m s}
$$

with

$$
a_{m}\left(\gamma_{j} P\right)=\sum_{\substack{m_{1}+m_{2}=m \\\left(M_{1}, M_{2}\right) \in \mathbb{A}_{m_{1}}^{+} \times \mathbb{A}_{m_{2}}^{+}}} \chi_{\gamma_{j} P}\left(M_{1} M_{2}^{2}\right)
$$

By [6, Lemma 2.3], we have

$$
\left|a_{m}\left(\gamma_{j} P\right)\right| \leq\left(\begin{array}{c}
2 n-2 \\
m
\end{array}\right) q^{\frac{m}{2}}
$$

so

$$
\left|\sum_{m=n}^{2 n-2} a_{m}\left(\gamma_{j} P\right) q^{-m s}\right| \leq 2^{2 n-2}\left(q^{\frac{1}{2}-\sigma}\right)^{n}\left(1-q^{\frac{1}{2}-\sigma}\right)^{-1}
$$

for $s \in \mathbb{C}$ with $\sigma=\operatorname{Re}(s)>1 / 2$. Hence, we have

$$
\begin{array}{r}
\left|\frac{1}{\# \mathcal{P}_{n}(\mathbb{A})} \sum_{P \in \mathcal{P}_{n}(\mathbb{A})} \sum_{m=n}^{2 n-2} a_{m}\left(\gamma_{j} P\right) q^{-m s}\right| \\
\leq 2^{2 n-2}\left(q^{\frac{1}{2}-\sigma}\right)^{n}\left(1-q^{\frac{1}{2}-\sigma}\right)^{-1} .
\end{array}
$$


Now, we consider

$$
\begin{aligned}
\mathcal{Z}_{n, j}^{\prime}(s) & =\frac{1}{\# \mathcal{P}_{n}(\mathbb{A})} \sum_{m=0}^{n-1} \sum_{P \in \mathcal{P}_{n}(\mathbb{A})} a_{m}\left(\gamma_{j} P\right) q^{-m s} \\
& =\frac{1}{\# \mathcal{P}_{n}(\mathbb{A})} \sum_{m=0}^{n-1} \sum_{\substack{m_{1}+m_{2}=m \\
\left(M_{1}, M_{2}\right) \in \mathbb{A}_{m}^{+} \times \mathbb{A}_{m}^{+}}} \sum_{P \in \mathcal{P}_{n}(\mathbb{A})} \chi_{\gamma_{j} P}\left(M_{1} M_{2}^{2}\right) q^{-m s} .
\end{aligned}
$$

Write $\mathcal{Z}_{n, j}^{\prime}(s)=\alpha_{n, j}(s)+\beta_{n, j}(s)$ with

$$
\alpha_{n, j}(s)=\frac{1}{\# \mathcal{P}_{n}(\mathbb{A})} \sum_{m=0}^{n-1} \sum_{\substack{m_{1}+m_{2}=m \\\left(M_{1}, M_{2}\right) \in \mathbb{A}_{m}^{+} \times \mathbb{A}_{m}^{+} \\ M_{1} M_{2}^{2}: \operatorname{not} \text { cube }}} \sum_{P \in \mathcal{P}_{n}(\mathbb{A})} \chi_{\gamma_{j} P}\left(M_{1} M_{2}^{2}\right) q^{-m s}
$$

and

$$
\beta_{n, j}(s)=\frac{1}{\# \mathcal{P}_{n}(\mathbb{A})} \sum_{m=0}^{n-1} \sum_{\substack{m_{1}+m_{2}=m \\\left(M_{1}, M_{2}\right) \in \mathbb{A}_{m}^{+} \times \mathbb{A}_{m}^{+} \\ M_{1} M_{2}^{2}: \text { cube }}} \sum_{P \in \mathcal{P}_{n}(\mathbb{A})} \chi_{\gamma_{j} P}\left(M_{1} M_{2}^{2}\right) q^{-m s} .
$$

If $M_{1} M_{2}^{2}$ is not cube, by (3.6), we have

$$
\left|\sum_{P \in \mathcal{P}_{n}(\mathbb{A})} \chi_{\gamma_{j} P}\left(M_{1} M_{2}^{2}\right)\right| \leq 2 q^{\frac{n}{2}} .
$$

For $s \in \mathbb{C}$ with $\sigma=\operatorname{Re}(s)>\frac{1}{2}$, using (3.9) and the fact that $\# \mathcal{P}_{n}(\mathbb{A})>$ $\frac{q^{n}}{2 n}$, we have

$$
\begin{aligned}
\left|\alpha_{n, j}(s)\right| & \leq \frac{1}{\# \mathcal{P}_{n}(\mathbb{A})} \sum_{m=0}^{n-1} \sum_{\substack{m_{1}+m_{2}=m \\
\left(M_{1}, M_{2}\right) \in \mathbb{A}_{m}^{+} \times \mathbb{A}_{m}^{+} \\
M_{1} M_{2}^{2}: \operatorname{not} \operatorname{cube}}}\left|\sum_{P \in \mathcal{P}_{n}(\mathbb{A})} \chi_{\gamma_{j} P}\left(M_{1} M_{2}^{2}\right)\right| q^{-m s} \\
& <4 n q^{-\frac{n}{2}} \sum_{m=0}^{n-1} \sum_{\substack{m_{1}+m_{2}=m \\
\left(M_{1}, M_{2}\right) \in \mathbb{A}_{m}^{+} \times \mathbb{A}_{m}^{+}}} q^{-m \sigma} \\
& <4 n q^{-\frac{n}{2}}\left(\frac{1-q^{n(1-\sigma)}\left(1+n-n q^{(1-\sigma)}\right)}{\left(1-q^{(1-\sigma)}\right)^{2}}\right) \rightarrow 0
\end{aligned}
$$


as $n \rightarrow \infty$. Now, we consider $\beta_{n, j}(s)$. Since $P \nmid M_{1} M_{2}$ and $M_{1} M_{2}^{2}$ is cube, we have $\chi_{\gamma_{j} P}\left(M_{1} M_{2}^{2}\right)=1$. Hence, we have

$$
\beta_{n, j}(s)=\sum_{\substack{m_{1}+m_{2}=n \\\left(M_{1}, M_{2}\right) \in \mathbb{A}_{m}^{+} \times \mathbb{A}_{m_{2}}^{+} \\ M_{1} M_{2}^{2}: \text { cube }}}\left|M_{1}\right|^{-s}\left|M_{2}\right|^{-s}
$$

Put

$$
L(s)=\sum_{\substack{\left(M_{1}, M_{2}\right) \in \mathbb{A}^{+} \times \mathbb{A}^{+} \\ P \nmid M_{1} M_{2}, M_{1} M_{2}^{2}: \text { cube }}}\left|M_{1}\right|^{-s}\left|M_{2}\right|^{-s} .
$$

Then, as in $[6, \S 2]$, we have

$$
L(s)=\frac{\zeta_{\mathbb{A}}(3 s)^{2} \zeta_{\mathbb{A}}(2 s)}{\zeta_{\mathbb{A}}(6 s)}
$$

and

$$
\left|\beta_{n, j}(s)-L(s)\right| \leq C n^{2} q^{\frac{n}{3}(1-3 \sigma)}
$$

for $\sigma=\operatorname{Re}(s)>\frac{1}{3}$ and some constant $C$ which depends on $s$ but is independent of $n$. By (3.10) and (3.11), we have that for $\sigma=\operatorname{Re}(s)>\frac{1}{3}$,

$$
\mathcal{Z}_{n, j}^{\prime}(s)=\frac{\zeta_{\mathbb{A}}(3 s)^{2} \zeta_{\AA}(2 s)}{\zeta_{\mathbb{A}}(6 s)}+O\left(C n^{2} q^{\frac{n}{3}(1-3 \sigma)}\right) .
$$

Since $C n^{2} q^{-\frac{2 n}{3}}=o\left(4^{n} q^{-\frac{n}{2}}\right)$, by (3.8) and (3.12), we have

$$
\mathcal{Z}_{n, j}(1)=\frac{1}{\# \mathcal{P}_{n}(\mathbb{A})} \sum_{P \in \mathcal{P}_{n}(\mathbb{A})} \prod_{i=1}^{2} L\left(1, \chi_{\gamma_{j} P}^{i}\right)=\frac{\zeta_{\mathbb{A}}(2) \zeta_{\mathbb{A}}(3)^{2}}{\zeta_{\mathbb{A}}(6)}+O\left(4^{n} q^{-\frac{n}{2}}\right) .
$$

Let $P \in \mathcal{P}_{n}(\mathbb{A})$. By (3.1), we have that if $3 \nmid n$,

$$
\prod_{i=1}^{2} L\left(1, \chi_{\gamma_{j} P}^{i}\right)=q^{1-n} h\left(\mathcal{O}_{k\left(\sqrt[3]{\gamma_{j} P}\right)}\right) \quad(0 \leq j \leq 2)
$$

and, if $3 \mid n$,

$$
\begin{aligned}
\prod_{i=1}^{2} L\left(1, \chi_{P}^{i}\right) & =q^{-n}(q-1)^{2} h\left(\mathcal{O}_{k(\sqrt[3]{P})}\right) R\left(\mathcal{O}_{k(\sqrt[3]{P})}\right) \\
& =\frac{q^{-n+2}}{\zeta_{\mathbb{A}}(2)^{2}} h\left(\mathcal{O}_{k(\sqrt[3]{P})}\right) R\left(\mathcal{O}_{k(\sqrt[3]{P})}\right)
\end{aligned}
$$




$$
\begin{aligned}
\prod_{i=1}^{2} L\left(1, \chi_{\gamma_{j} P}^{i}\right) & =\frac{q^{-n}\left(q^{3}-1\right)}{3(q-1)} h\left(\mathcal{O}_{k\left(\sqrt[3]{\gamma_{j} P}\right)}\right) \\
& =\frac{\zeta_{\mathbb{A}}(2) q^{-n+2}}{3 \zeta_{\mathbb{A}}(4)} h\left(\mathcal{O}_{k\left(\sqrt[3]{\gamma_{j} P}\right)}\right) \quad(j=1,2) .
\end{aligned}
$$

As $n \rightarrow \infty$ with $3 \nmid n$, by (3.2), (3.7) and (3.13), we have

$$
\frac{1}{\# \mathcal{A}_{n}} \sum_{K \in \mathcal{A}_{n}} h\left(\mathcal{O}_{K}\right)=\frac{\zeta_{\mathbb{A}}(2) \zeta_{\mathbb{A}}(3)^{2}}{\zeta_{\mathbb{A}}(6)} q^{n-1}+O\left(4^{n} q^{\frac{n}{2}}\right),
$$

and $n \rightarrow \infty$ with $3 \mid n$, by (3.3), (3.4), (3.7), (3.14) and (3.15), we have

$$
\begin{aligned}
\frac{1}{\# \mathcal{B}_{n}} \sum_{K \in \mathcal{B}_{n}} h\left(\mathcal{O}_{K}\right) R\left(\mathcal{O}_{K}\right) & =\frac{\zeta_{\mathbb{A}}(2)^{3} \zeta_{\mathbb{A}}(3)^{2}}{\zeta_{\mathbb{A}}(6)} q^{n-2}+O\left(4^{n} q^{\frac{n}{2}}\right), \\
\frac{1}{\# \mathcal{C}_{n}} \sum_{K \in \mathcal{C}_{n}} h\left(\mathcal{O}_{K}\right) & =\frac{3 \zeta_{\mathbb{A}}(3)^{2} \zeta_{\mathbb{A}}(4)}{\zeta_{\mathbb{A}}(6)} q^{n-2}+O\left(4^{n} q^{\frac{n}{2}}\right) .
\end{aligned}
$$

\section{References}

[1] S. Bae, H. Jung, and P-L. Kang, Artin-Schreier extensions of the rational function field, Math. Z. 276 (2014), 613-633.

[2] Y.-M. J. Chen, Average values of L-functions in characteristic two, J. Number Theory 128 (7) (2008), 2138-2158.

[3] J. Hoffstein and M. Rosen, Average values of L-series in function fields, J. Reine Angew. Math. 426 (1992), 117-150.

[4] C.-N. Hsu, On certain character sums over $\mathbb{F}_{q}[T]$, Proc. Amer. Math. Soc. 126 (3) (1998), 647-652.

[5] R. Prime, Averaging imaginary prime quadratic L-series, Preprint.

[6] M. Rosen, Average value of class numbers in cyclic extensions of the rational function field, Number theory (Halifax, NS, 1994), CMS Conf. Proc., vol. 15, Amer. Math. Soc., Providence, RI, 1995, pp. 307-323.

Hwanyup Jung

Department of Mathematics Education

Chungbuk National University

Cheongju 361-763, Korea

E-mail: hyjung@chungbuk.ac.kr 